Innovación y Desarrollo Tecnológico y Social (2021) 3 (1): 45-88

\title{
IARespira: experiencias en el diseño y desarrollo de un Ventilador Mecánico No Invasivo para COVID-19
}

\author{
Martín Salibe 1; Elias S. Fliger'; Leandro García ${ }^{\text {; }}$ Gustavo E. Romero 1,2,3 \\ ${ }^{1}$ Instituto Argentino de Radioastronomía (IAR), CONICET-CIC-UNLP, Buenos Aires, Argentina, \\ ${ }^{2}$ Facultad de Ciencias Astronómicas y Geofísicas (FCAyG), Universidad Nacional de La Plata, La \\ Plata, Buenos Aires, Argentina, ${ }^{3}$ gustavo.esteban.romero@gmail.com
}

Resumen. La irrupción de la pandemia de COVID-19 en febrero de 2020 y la velocidad de propagación entre la población a nivel mundial de la enfermedad, provocó una demanda inusitada de ventiladores mecánicos, producto de los efectos reactivos al virus en el sistema respiratorio de las personas. El acelerado incremento de pacientes infectados graves, sobre exigió a los servicios de atención médica causando un colapso general del sistema de salud. El área de Transferencia de Tecnología del Instituto Argentino de Radioastronomía (IAR) reaccionó al problema iniciando el diseño y desarrollo de un ventilador mecánico no invasivo (VMNI) de bajo costo, denominado IARespira, para la asistencia a pacientes de gravedad moderada, durante el brote de COVID-19. EI VMNI trabaja con un control de presión con tres modos de ventilación posibles: CPAP (presión positiva continua en las vías respiratorias), BiPAP (presión positiva de dos niveles) y Asistida/Controlada. Además, el proyecto propone una solución a la dificultad de acceso a insumos críticos con elementos disponibles principalmente en el mercado local. El diseño se orientó para una rápida fabricación y pronta disponibilidad en los centros de salud de la Argentina.

Palabras claves: ventilación mecánica; diseño; equipamiento médico; COVID-19. 
Innovación y Desarrollo Tecnológico y Social (2021) 3 (1): 45-88

\section{IARespira design and development experiences of a Non-Invasive Mechanical} Ventilator for COVID-19

Abstract. The surge of COVID-19 in February 2020 and its spread among the population worldwide, caused an unusual demand of mechanical ventilators due to the effects of the disease on the respiratory system of the patients. The rapid growth of acute cases stressed care units and menaced to produce the general collapse of health systems. In response to such a situation, the Technology Transfer area of the Argentine Institute of Radio Astronomy (IAR), initiated the design and development of a non-invasive mechanical ventilator (NIMV or NIV), so-called IARespira, for the treatment of moderate COVID-19 patients during the outbreak. The NIV works as a pressure control device with three ventilation modes: CPAP, BiPAP and Assisted/Controlled. The device was designed taking into account the availability restrictions to critical resources, mainly in the local market. Its design was oriented towards a rapid manufacture and distribution to the several health centers across Argentina.

Keywords: mechanical ventilation; design; medical equipment; COVID-19.

\section{Novedad u originalidad local en el conocimiento}

La sobrecarga del sistema de salud mundial, al inicio de la crisis sanitaria ocasionada por el COVID-19 produjo una demanda inusitada de suministros médicos para atender a pacientes con insuficiencia respiratoria aguda. Entre los dispositivos complejos más requeridos, el ventilador mecánico resultó ser una pieza clave para dar respuesta al nuevo virus.

Numerosas iniciativas alrededor del mundo buscaron diversas formas de abordar la escasez de ventiladores mecánicos. Estas abarcaron desde emprendimientos en la comunidad open-source hasta asociaciones público-privadas, que diseñaron prototipos y mecanismos para facilitar la provisión de oxígeno a pacientes graves. 
Innovación y Desarrollo Tecnológico y Social (2021) 3 (1): 45-88

Se encontraron variaciones del MIT Emergency Ventilator (MIT E-Vent), entre los diseños más frecuentes, basados en la bolsa de resucitación manual o AMBU (Airway Mask Bag Unit, siglas de la marca comercial) como herramienta de emergencia frente al COVID-19.

El aporte del IAR se centró en un diseño alternativo propio, de escasa exploración a nivel local, basado en una turbina de aire, como soporte intermitente de pacientes moderados a graves, mediante la ventilación mecánica no invasiva. Se incluyó un sistema de control inteligente desarrollado a partir de la experiencia previa del grupo de trabajo en esa clase de tecnologías. Se definió un dispositivo de ventilación en presión control y 3 modos de ventilación posibles: CPAP (presión positiva continua en las vías respiratorias), SIMV-PCIPS (nombre comercial BiPAP, presión positiva de dos niveles) y un modo Asistido/Controlado, A/C (modo controlado por presión que combina respiraciones mandatorias regulares e inspiraciones asistidas cuando el paciente hace un esfuerzo inspirador), según el esfuerzo respiratorio que presente el paciente al momento de su evaluación médica.

\section{Grado de relevancia}

Los ventiladores mecánicos, en conjunto con oxímetros de pulso, son piezas clave para tratar a pacientes con enfermedades respiratorias. En particular, las complicaciones derivadas de la infección con SARS-CoV-2, como el síndrome de dificultad respiratoria aguda (SDRA). 
La propagación exponencial del nuevo virus, sumado a la dinámica de la población mundial, provocó, entre enero y abril de 2020, el desborde simultáneo de las unidades de cuidados intensivos (UCI) en lugares tan distantes como China, España, Italia y Ecuador. La abrupta demanda de ventiladores en combinación con una producción limitada a condiciones nominales y la puja entre los estados económicamente más solventes, desencadenó la falta generalizada de equipamiento médico para tratar el COVID-19.

Instituciones, empresas, comunidades y particulares orientaron sus esfuerzos al diseño y desarrollo de soluciones de emergencia para proveer el equipamiento necesario. Sólo en nuestro país, se contabilizaron alrededor de 50 proyectos basados mayoritariamente en el modelo E-Vent, vinculados a ventiladores mecánicos, con diferentes niveles de complejidad, grado de avance, eficacia y seguridad. En ese sentido, el diseño de IARespira sumó a los emprendimientos existentes un esquema diferente de funcionamiento y operación: basado en turbina de aire, compacto, de uso simple, bajo costo y ventilación no invasiva (VNI).

\section{Grado de pertinencia}

El IAR adoptó una postura activa frente a la pandemia, analizando y discutiendo la situación, mediante la coordinación del área de Vinculación y Transferencia Tecnológica (VTT) del Instituto. De esta manera, se conformó un equipo de trabajo colaborativo y multidisciplinario entre el personal científico y tecnológico del IAR, 
Innovación y Desarrollo Tecnológico y Social (2021) 3 (1): 45-88

docentes y alumnos de la UNLP, bioingenieros, empresas de equipamiento médico, médicos especialistas y particulares.

Se plantearon dos líneas de trabajo claramente definidas. La primera, orientada al tratamiento de pacientes afectados por el COVID-19 y, la segunda, destinada a la desinfección de espacios públicos, para minimizar los riesgos de contagio de las personas.

El Instituto cuenta con más de 20 años de experiencia en el desarrollo de tecnología aplicada al segmento aeroespacial. La experiencia en esta área crítica nos permitió incursionar en el sector médico, forjando vínculos con empresas, hospitales y grupos de investigación durante los últimos 6 años.

Además, contamos con tres departamentos técnicos con un claro impacto productivo:

- $\quad$ Sistemas informáticos, para la gestión de la infraestructura IT y desarrollo de software;

- $\quad$ Electrónica, para el diseño y desarrollo de hardware y firmware crítico;

- $\quad$ Mecánico para el diseño y fabricación de dispositivos y equipamiento.

\section{Grado de demanda}

El único aporte monetario que recibió el proyecto IARespira fue otorgado por el CCT de La Plata, al inicio del ASPO (Aislamiento Social Preventivo y Obligatorio). Además, 
Innovación y Desarrollo Tecnológico y Social (2021) 3 (1): 45-88

sumó la colaboración de terceros, quienes aportaron su tiempo al desarrollo del VMNI.

Estas contribuciones permitieron desarrollar los primeros modelos y prototipos para la validación de los diseños y difusión del trabajo en medios informativos y empresas. De esta manera, la empresa Electromedik S.A. sumó materiales y personal para discutir los modelos y el desarrollo del producto.

El proyecto logró amplia difusión en los medios y fue declarado de interés municipal en julio de 2020. Entre las menciones al proyecto IARespira en los medios, se puede mencionar a TV Universidad (2020), ECO medios (2020), BA Productivo (2020), Diario Perfil (2020), Agencia Télam (2020), Radio Nacional (2020), 0221.com.ar (2020), IN Noticias (2020), Diario El Día (2020), América TV (2020), Investiga UNLP (2020), IAR Extensión (2020).

\section{Desarrollo del producto}

El proyecto del Ventilador Mecánico No Invasivo (VMNI), IARespira, tomó como referencia algunas de las iniciativas que estaban siendo desarrollados para cubrir los déficits de equipos de asistencia respiratoria, ubicando el objetivo en el diseño de un equipo cuya relación costo-beneficio resulte conveniente para una rápida implementación y distribución hacia los centros de salud (Al Husseini et al., 2010). En cuanto al principio de funcionamiento, se definió un dispositivo de ventilación en presión control (PC) de presión positiva (PPV) y 3 modos de ventilación posibles: 
Innovación y Desarrollo Tecnológico y Social (2021) 3 (1): 45-88

CPAP (presión positiva continua en las vías respiratorias), SIMV-PCIPS (nombre comercial BiPAP, presión positiva en las vías aéreas de dos niveles) y un modo de respiración Asistida/Controlada, A/C (modo controlado por presión que combina respiraciones mandatorias regulares e inspiraciones asistidas cuando el paciente hace un esfuerzo inspirador), según el esfuerzo respiratorio que presente el paciente al momento de su evaluación médica.

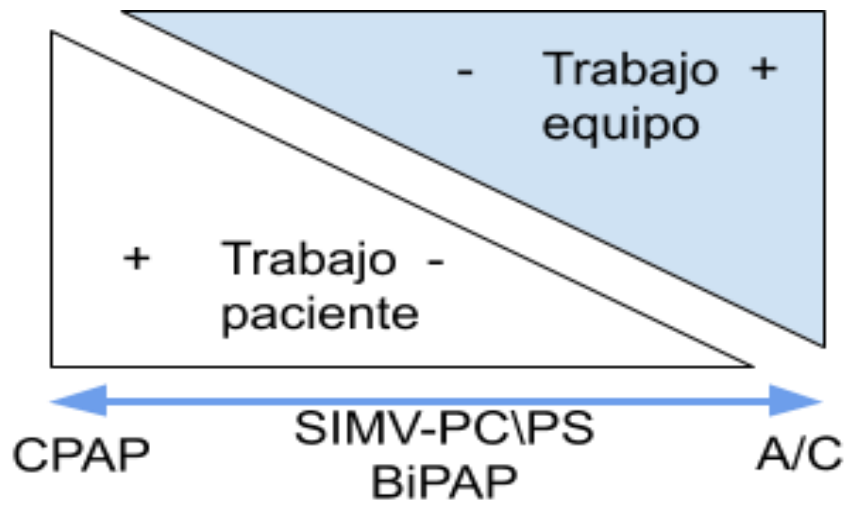

Figura 1: Uso de modo ventilatorio en función del esfuerzo del paciente y del ventilador.

Figure 1: Ventilation use mode as a function of the patient's and ventilator's effort.

El dispositivo puede ser conectado a la línea de mezcla presurizada de oxígeno y aire, externa al equipo, para suministrar al paciente diferentes concentraciones de $\mathrm{O}_{2}$. La regulación de presión se provee mediante la combinación de una turbina y la entrada de $\mathrm{O}_{2}$, que inyecta un flujo variable de la mezcla de aire y $\mathrm{O}_{2}$ en función del perfil de presión configurado para el paciente. 
Innovación y Desarrollo Tecnológico y Social (2021) 3 (1): 45-88

Dadas las exigencias impuestas por la normativa que regula los equipos médicos, particularmente sobre los aspectos de software, hemos aproximado la solución mediante diferentes bloques electrónicos:

- $\quad$ Configuración de las variables de referencia y límites de alarmas para la presión y temporización de los ciclos respiratorios.

- Control PID sobre la variable presión.

- $\quad$ Circuito de supervisión, monitoreo de las variables y disparo de alarmas.

- Acondicionamiento de las entradas analógicas y adaptación de las entradas/salidas discretas.

La lógica de supervisión y la de configuración y control se encuentran físicamente separadas en microcontroladores dedicados para cada funcionalidad, con el objetivo de aumentar la robustez del sistema.

En definitiva, la filosofía de diseño se orientó según las siguientes características:

Bajo costo.

Conjunto reducido de partes y componentes.

Operación sencilla.

Complejidad reducida.

Fácil fabricación y distribución. 
Innovación y Desarrollo Tecnológico y Social (2021) 3 (1): 45-88

\section{Características y prestaciones}

El dispositivo propuesto por el IAR se basa inicialmente en el prototipo detallado en (Lee, 2020) y proyectos más evolucionados como en ACUTE-19 (2020), Garmendia et al. (2020), Makers-for-life (2020), algunos de los cuales ya se encuentran en fase de ensayos clínicos o aprobados para uso médico.

El equipo se realimenta de las señales de flujo y presión, para actuar sobre la turbina y electroválvulas, al mismo tiempo que monitorea las diferentes variables que podrían disparar las alarmas correspondientes, en caso de detectar alguna falla. Luego, un panel de configuración, como interfaz de usuario, permite establecer los principales parámetros operacionales y la visualización de las variables vitales críticas, que deberán ser evaluadas por el profesional a cargo. En la Figura 2, puede verse un esquema del diseño descrito.

Un punto central para destacar es que, pese a la sencillez del concepto, el VMNI expuesto busca mantener un alto grado de confiabilidad y robustez, orientado al ambiente exigente y crítico en el que podría operarse el equipo. La forma de resolver el problema ha sido implementando un procedimiento iterativo de revisión entre los miembros del equipo de desarrollo, sumado a la visión de especialistas en materia médica. Desde el punto de vista de la electrónica, se definió un circuito de supervisión con el propósito de procesar las variables del ventilador, y accionar las alarmas sonoras y visuales, en caso de detectar alguna anomalía. 
Innovación y Desarrollo Tecnológico y Social (2021) 3 (1): 45-88

Los componentes detallados son, en su mayoría, de calidad médica (tubos, mascarillas, filtros, turbina, etc.) y otros de uso industrial (uC, fuente de alimentación, display, LCD, electroválvulas, etc.) de amplia disponibilidad en el mercado local y con múltiples proveedores. Esta decisión de diseño se funda en la imposibilidad de acceder normalmente a los insumos y partes necesarias para construir cada unidad. Es un punto particularmente complejo para las empresas del sector médico, que tiene su capacidad de producción seriamente limitada, debido a la enorme demanda generada por el resto de los países en similar situación. El despliegue general de la Figura 2 muestra los principales componentes necesarios para la implementación del VMNI IARespira.

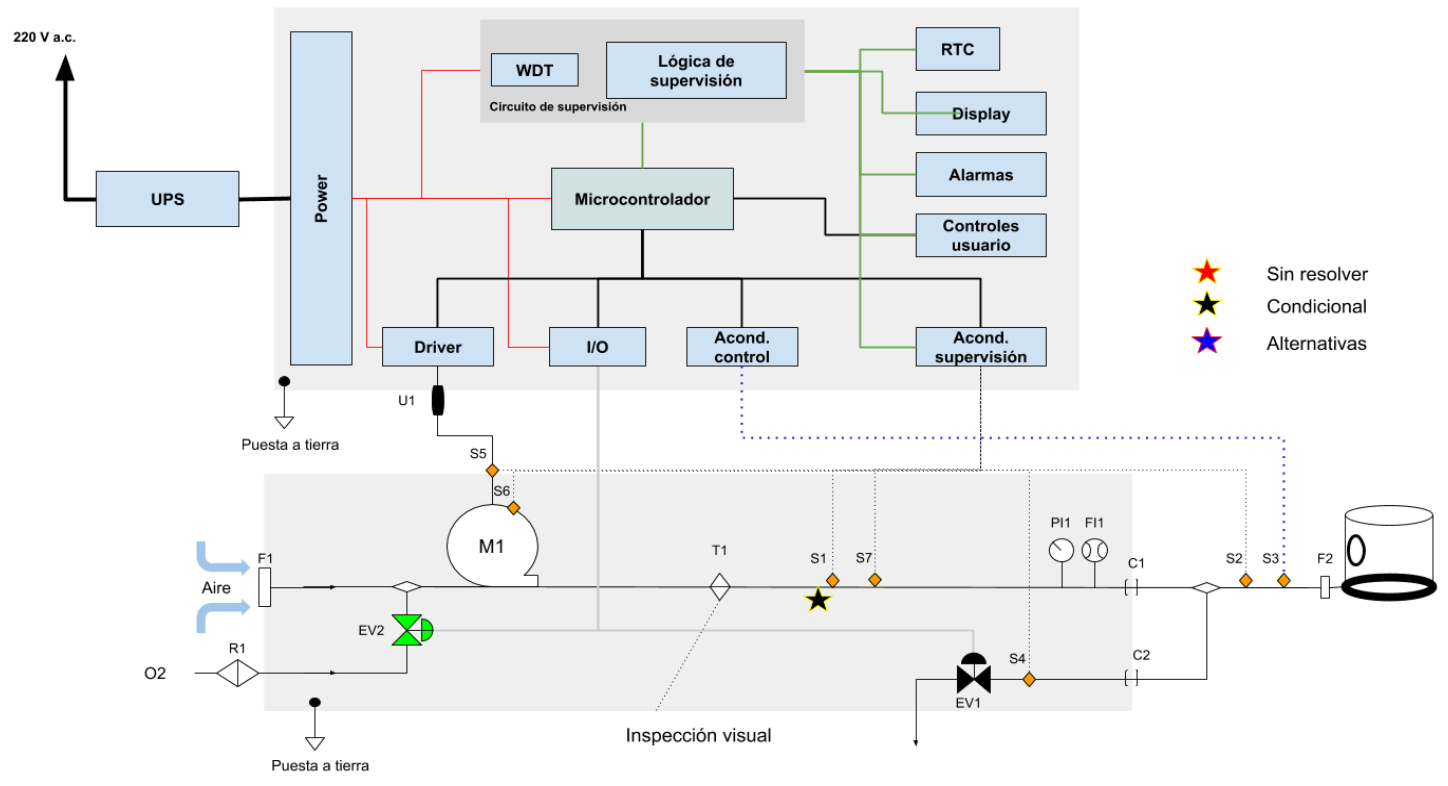

Figura 2: Descripción del conexionado y hardware necesario para implementar una solución de bajo costo de VMNI.

Figure 2: Description of wiring and hardware needed to provide a low-cost NIV solution. 
Innovación y Desarrollo Tecnológico y Social (2021) 3 (1): 45-88

Descripción de los módulos

\section{Módulos electrónicos}

El módulo electrónico que aloja a la placa del microcontrolador (Circuito de supervisión) (Pennisi, 2020), controlador y periféricos, incluido driver del forzador de aire, está protegido mediante láminas para absorción de EMI, para evitar interferencias desde y hacia otros equipos médicos.

\section{Alimentación eléctrica}

La distribución desde la línea de red de 220 V c.a. se realiza desde una fuente UPS, con capacidad mínima de 1 kVA. La tensión regulada alimenta luego a una fuente de PC ATX, desde donde se proveen las tensiones a los diferentes circuitos electrónicos.

\section{Circuito de supervisión}

Centrado en una placa Raspberry Pi 3, para la supervisión, monitoreo de las variables y disparo de alarmas. La detección de cualquier parámetro fuera de rango y/o señal de alarma, activa la alarma sonora y visual correspondiente a la falla detectada.

\section{Alarmas}

Se establecen una serie de alarmas visuales y sonoras que permiten identificar las posibles fallas detectadas y orientar al usuario, especialmente en el caso de riesgo de vida del paciente (Thomas y Galvin, 2008). Las fallas pueden ser informadas mediante indicadores luminosos, mensaje en el display y chicharra sonora de 
manera intermitente hasta $5 \mathrm{~m}$ de distancia, siguiendo las recomendaciones de SATI (2020).

Tabla 1: Listado de alarmas.

Table 1: List of alarms.

\begin{tabular}{|c|c|}
\hline Tipo & Detalle de alarma \\
\hline 1. Alimentación de UPS & $\begin{array}{l}\text { a. Carga de batería baja. } \\
\text { b. Corte red de alimentación } 220 \text { V a.c. } \\
\text { c. Fallo de regulación de salida. } \\
\text { d. Tiempo remanente }<15 \text { min. }\end{array}$ \\
\hline 2. Turbina & $\begin{array}{l}\text { a. Exceso de corriente. } \\
\text { b. Alta temperatura }\left(>85^{\circ} \mathrm{C}\right) \text {. }\end{array}$ \\
\hline 3. Presión & $\begin{array}{l}\text { a. Presión pico > } 40 \text { cmH20. } \\
\text { b. Presión insp < límite bajo. } \\
\text { c. PEEP > límite alto. } \\
\text { d. PEEP < límite bajo. } \\
\text { e. Presión espiración > límite alto. }\end{array}$ \\
\hline 4. Flujo & $\begin{array}{l}\text { a. Pico de flujo inspirado. } \\
\text { b. Fuga de aire. }\end{array}$ \\
\hline 5. Tiempo & $\begin{array}{l}\text { a. Frecuencia respiratoria < } 10 \mathrm{rpm} \text {. } \\
\text { b. Frecuencia respiratoria }>30 \mathrm{rpm} \text {. } \\
\text { c. Tiempo inspiración }>1,2 \mathrm{~s} \text {. } \\
\text { d. Tiempo inspiración }<0,7 \mathrm{~s} \text {. }\end{array}$ \\
\hline 6. Volumen & $\begin{array}{l}\text { a. Volumen inspiratorio }>700 \mathrm{ml} \text { o } 110 \% \text { del } \\
\text { configurado. } \\
\text { b. Volumen inspiratorio }<300 \mathrm{ml} \text { o } 90 \% \text { del configurado. } \\
\text { c. Volumen minuto máximo. }\end{array}$ \\
\hline 7. Configuración & $\begin{array}{l}\text { a. Seteo de Pmax }>60 \mathrm{cmH} 2 \mathrm{O} \text {. } \\
\text { b. PEEP > límite alto. } \\
\text { c. Frecuencia respiratoria > límite alto. } \\
\text { d. Frecuencia respiratoria < límite bajo. } \\
\text { e. Tiempo inspiración }>1,2 \mathrm{~s} \text {. } \\
\text { f. Tiempo inspiración }<0,7 \mathrm{~s} \text {. }\end{array}$ \\
\hline 8. Supervisión & $\begin{array}{l}\text { a. Límite de vida útil turbina }<500 \text { hs. } \\
\text { b. Tiempo operativo acumulado }>20.000 \text { hs. }\end{array}$ \\
\hline
\end{tabular}


Innovación y Desarrollo Tecnológico y Social (2021) 3 (1): 45-88

\section{Teclado y panel de control}

El equipo cuenta con un teclado, para el seteo de parámetros, consulta del estado general del sistema y visualización de las diferentes variables. Este debe ser provisto para una sencilla operación del usuario con vestimenta de protección.

\section{Display}

Pequeña pantalla o visor de LCD para informar al usuario del estado general del sistema, la evolución de las variables y seteo de los parámetros de operación.

Principales parámetros para visualizar:

- Seteos:

- Frecuencia respiratoria [rpm].

- Tiempo inspiratorio [s].

○ $\quad$ Relación I:E [I:E].

- Presión pico [cmH2O].

- PEEP $[\mathrm{cmH} 2 \mathrm{O}]$.

- Modo de ventilación [Texto]

- Valores corrientes de:

- Frecuencia respiratoria [rpm].

- $\quad$ Frecuencia de respiraciones espontáneas [rpm].

- Frecuencia respiratoria total (automáticas + espontáneas) [rpm].

- Presión pico [cmH2O].

○ PEEP $[\mathrm{cmH} 2 \mathrm{O}]$. 
Innovación y Desarrollo Tecnológico y Social (2021) 3 (1): 45-88

- $\quad$ Presión promedio en las vías aéreas [cmH2O].

- Volumen inspirado [ml].

- Volumen minuto inspirado [l/min].

- $\quad$ Fracción de $\mathrm{O}_{2}[\% \mathrm{~V} / \mathrm{V}]$.

$\circ \quad$ Fugas $[\mathrm{ml} / \mathrm{min}]$.

- $\quad$ Monitor de uso

- Alarmas presentes activas.

- Tiempo en actividad con paciente [hs].

- Fecha y hora de inicio ventilación [AAA-MM-DD hh:mm:ss].

- Tiempo de actividad acumulada [hs].

- Identificación del equipo

- Fabricante [Texto].

- Modelo [Texto].

- Versión de software alarmas [Texto].

\section{Driver de la turbina}

El motor de la turbina o forzador de aire requiere de un driver para controlar su funcionamiento durante cualquiera de los modos de operación del equipo. Un motor típico para estas aplicaciones es el BLDC, utilizados ampliamente en la industria.

\section{Turbina forzadora de aire}

Tensión de alimentación máxima de 24 V, 43 W de consumo y hasta 40.000 rpm. 
Innovación y Desarrollo Tecnológico y Social (2021) 3 (1): 45-88

\section{Drivers de electroválvulas}

Accionan las electroválvulas ON-OFF, presentes en el diseño del VMNI.

\section{Acondicionamiento}

Bloque compuesto por placas heterogéneas, integradas de manera tal que puedan compatibilizar con las entradas y salidas del uC.

\section{Comunicaciones}

Este módulo está sujeto al diseño final implementado y a la necesidad concreta durante la operación del equipo. No es esencial. Puede contener puerto de comunicación USB, RS-232, WIFI u otros.

\section{Sensor de $\mathrm{O}_{2}$}

Se seleccionó una celda de sensado de oxígeno, empleada para ventiladores y respiradores comerciales. La señal de S1 debe ser acondicionada para enviar al uC y mostrar el dato en el display. Salida analógica entre 0 y 5 V. Su disponibilidad se encuentra severamente limitada por la enorme demanda generada por la crisis sanitaria.

\section{Sensor de flujo inspiratorio}

Se analizaron alternativas para el sensor de flujo inspiratorio S2 y espiratorio S4. La primera opción es el sensor SFM3000-200C, de Sensirion. Sin embargo, la excesiva demanda de este producto dificulta su adquisición. Una alternativa es la medición de flujo mediante diferencias de presión con un sensor de presión diferencial. Un 
Innovación y Desarrollo Tecnológico y Social (2021) 3 (1): 45-88

proveedor nacional es especialista en el tema y se contactó para su implementación. Aún en estudio.

\section{Sensor de presión}

El sensor de presión proximal se encuentra definido por S3. Se trata de un sensor de presión diferencial de la serie 26PC, de Honeywell. El rango de medición es de +/1 psi (70,3cmH2O), acorde a los rangos de medición requeridos por la aplicación.

\section{Sensor de corriente}

El sensor de corriente $\mathrm{S5}$, en la entrada del motor forzador de aire, realimenta al uC para determinar el estado de funcionamiento. Se alimenta con $5 \mathrm{~V}$ y rango de medida de +/- 30 A, salida lineal en tensión.

\section{Electroválvula de admisión de $\mathrm{O}_{2}$ a presión}

La electroválvula ON-OFF de oxígeno, indicada PV1, permite el ingreso de oxígeno desde la línea de gas a presión hospitalaria o envasado. El ingreso de $\mathrm{O}_{2}$ deberá estar limitado por un regulador de presión a la entrada del equipo.

\section{Electroválvula de espiración}

La electroválvula ON-OFF de espiración, indicada PV2, permite la salida de los gases de los pulmones.

\section{Indicadores de presión y flujo mecánicos}

Se disponen dos indicadores mecánicos Fl y Pl, flujómetro y manómetro respectivamente, para ofrecer una redundancia externa al circuito electrónico. 
Innovación y Desarrollo Tecnológico y Social (2021) 3 (1): 45-88

\section{Módulos neumáticos}

\section{Filtros de aire}

Se disponen filtros de aire de entrada y de salida a modo de prefiltro, indicado como F1 y F3. Estos filtros pueden ser HEPA clase E12 o superior, disponibles ampliamente en el mercado.

El filtro F2 es del tipo HEMF, para conexión de los tubos de respiración, con diferentes proveedores locales, según normas nacionales e internacionales NIOSH (1996).

\section{Tubos}

Se emplean tubuladoras universales de respiración con doble rama, para conexiones de $22 \mathrm{~mm}$ de diámetro.

\section{Trampa de humedad}

En algunos casos es necesaria la incorporación de trampas de humedad para evitar acumulación de agua que pueda dañar los sensores y el equipo en general. Su incorporación no está definida en el diseño final.

\section{Entrada de $\mathrm{O}_{2}$}

Conexión de entrada de oxígeno para proveer al paciente.

\section{Conectores}

Conectores de $22 \mathrm{~mm}$ para conexión de tubuladoras. 
Innovación y Desarrollo Tecnológico y Social (2021) 3 (1): 45-88

\section{Modos ventilatorios}

IARespira cuenta con tres modos ventilatorios, típicamente usados en terapia de ventilación no invasiva (Ferrero, 2008): modo CPAP (de presión positiva continua), modo BiPAP (con dos niveles de presión positiva) y modo AC de respiración asistida/controlada (con presión controlada y respiraciones mandatorias regulares y asistidas ante un eventual esfuerzo inspiratorio). El uso de estos modos podría ser apropiado en terapias no invasivas para pacientes con síntomas de insuficiencia respiratoria (SADI, 2020, SEMUE, 2020). En los dos primeros modos el paciente debe poseer una respiración espontánea y estar consciente en todo momento. El tercer modo, también es usado en terapias de ventilación mecánica invasivas, en el cual es el ventilador quien comanda la respiración de forma regular al paciente (ciclo mandatorio), pero éste puede iniciar también un ciclo respiratorio mediante un esfuerzo inspiratorio reconocido por el ventilador (ciclo asistido).

\section{CPAP}

Este modo se denomina de presión positiva continua, porque el ventilador provee una presión constante (presión máxima, Pmax) en las vías aéreas. Dicha presión máxima es ajustable en el equipo. El paciente respira normalmente por sobre dicha presión.

\section{BIPAP}

En este modo, el ventilador provee dos niveles de presión. Un nivel de presión mínimo o PEEP (presión positiva al final de la espiración) y un nivel máximo que 
Innovación y Desarrollo Tecnológico y Social (2021) 3 (1): 45-88

denominamos presión máxima (en la literatura estos niveles se denominan en ocasiones EPAP e IPAP, respectivamente). En el caso del ventilador IARespira, cuando al final de una espiración el paciente hace un esfuerzo inspiratorio, la presión en máscara cae por debajo de un valor de presión umbral (Pumbral), el ventilador dispara un ciclo respiratorio, aumentando rápidamente la presión hasta alcanzar el valor Pmax. Esta presión se mantiene constante hasta que se cumple el tiempo de inspiración, Tinsp. Durante la espiración (Tesp), la presión desciende hasta el nivel PEEP de forma progresiva. Nótese, que Tesp no tiene por qué coincidir con el tiempo de espiración, que es determinado por el paciente, y que termina cuando comienza el siguiente esfuerzo de inspiración registrado por el sensor de presión.

\section{$A / C$}

En el modo A/C (respiración asistida/controlada), la inspiración está controlada por el ventilador tras ser ajustados los parámetros de presión máxima (Pmax), PEEP, tiempo de inspiración y frecuencia respiratoria (FR). El ventilador controla el número mínimo de respiraciones por minuto. Si el ventilador no detecta un esfuerzo inspiratorio por parte del paciente, transcurrido el periodo respiratorio (1/FR), el siguiente ciclo respiratorio será iniciado de manera mandatoria por el ventilador, de forma de cumplir con el número mínimo de respiraciones por minuto. En cambio si durante la espiración, el ventilador detecta un esfuerzo inspiratorio por parte del paciente (es decir, la presión desciende por debajo de un nivel de presión umbral, 
Innovación y Desarrollo Tecnológico y Social (2021) 3 (1): 45-88

menor que el valor de PEEP), el ventilador inicia un ciclo respiratorio de iguales características (Pmax, PEEP, Tinsp) que los "mandatorios".

\section{Esquema funcional del uC central}

El funcionamiento del VMNI depende de un bucle de control en tiempo real de todas las variables del sistema (tanto clínicas como operativas), lo cual permite ajustar el funcionamiento a la configuración del usuario (personal médico) y al requerimiento del paciente en forma automática. Este bucle de control se ejecuta de manera autónoma con el soporte de una placa de procesamiento central (microcontrolador), registrando las variables de entrada (Figura 3):

- $\quad$ Configuración del usuario por interfaz (personal médico).

- $\quad$ Configuración en memoria.

- $\quad$ Medición de presión.

- $\quad$ Medición de flujo.

- $\quad$ Medición de corriente.

- $\quad$ Medición de $\mathrm{O}_{2}$.

Y realizar el control sobre los dispositivos:

Turbina.

Electroválvulas. 


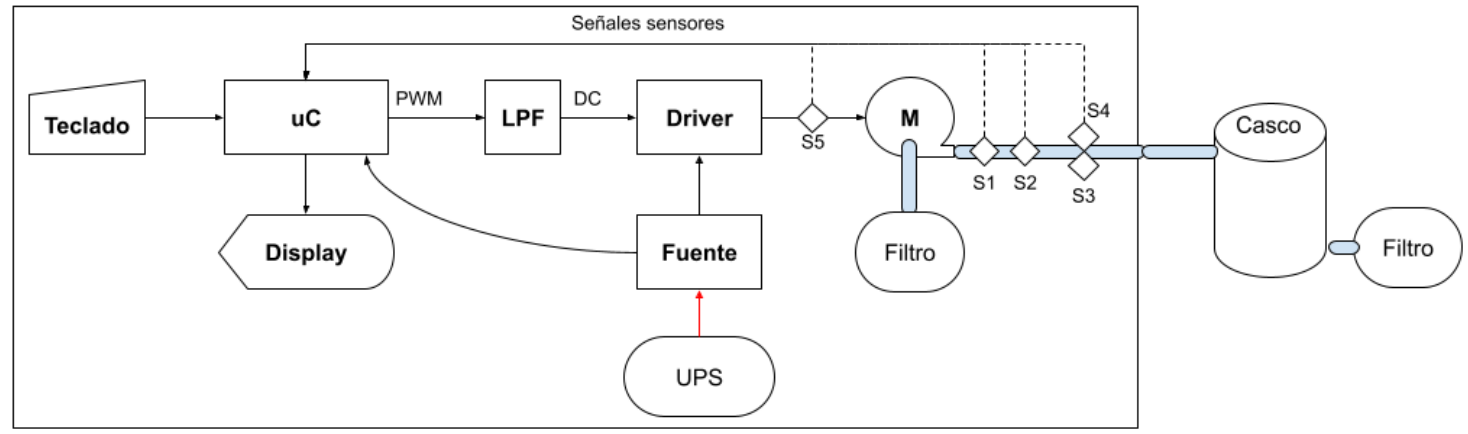

Figura 3: Diagrama de control y actuación del VMNI.

Figure 3: NIV control and actuating blocks diagram.

Además, el sistema muestra la información crítica para el usuario (variables clínicas y operativas), y también dispara las alarmas correspondientes. El monitoreo del sistema se lleva a cabo en forma local (display LCD), y se plantea la posibilidad de transmitir de forma remota (USB o red). En la Figura 4 se puede ver un diagrama simplificado del esquema funcional del sistema.

El esquema de la Figura 5, muestra el conexionado de los diferentes bloques funcionales del sistema y su relación en función de sus características:

- $\quad$ Entradas analógicas: adquisición de las variables sensadas.

- $\quad$ Entradas digitales: reloj de tiempo real (RTC), memoria y teclado.

- Salidas digitales: alarmas, display, watchdog (WDT), drivers, memoria y circuito de supervisión. 
Innovación y Desarrollo Tecnológico y Social (2021) 3 (1): 45-88

De esta forma, es posible mantener un elevado nivel de autonomía, al mismo tiempo que se incrementa el nivel de seguridad del sistema, separando los bloques de Gestión (uC central) y de Supervisión (uP de soporte), de manera independiente.

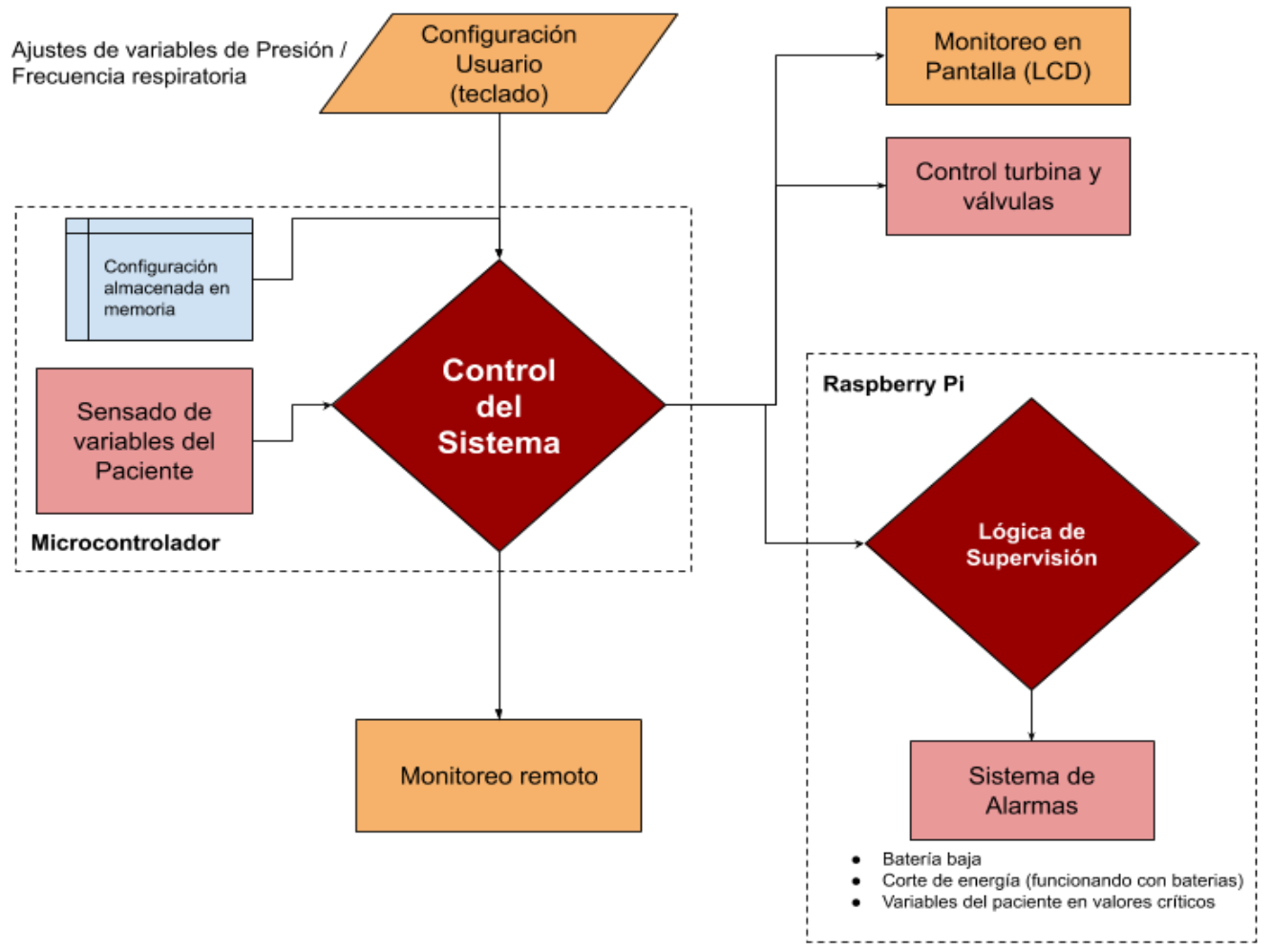

Figura 4: Diagrama de los sistemas de control y supervisión.

Figure 4: Control and supervisory systems diagram. 


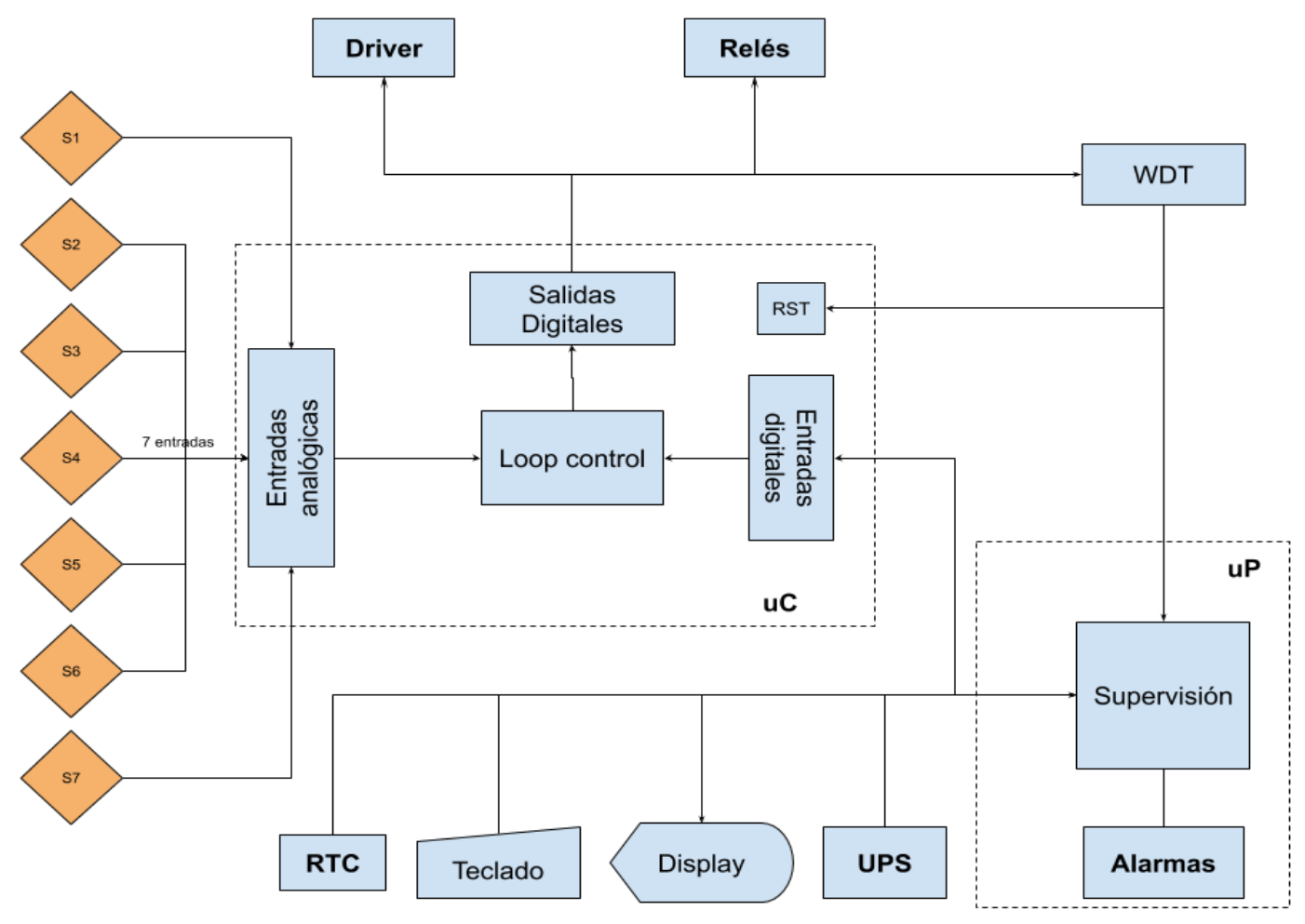

Figura 5: Bloques funcionales de control y supervisión.

Figure 5: Control and supervisory functional blocks.

\section{Esquema funcional del circuito de supervisión}

El circuito supervisor, de la Figura 6, se encarga de monitorear el estado general de la electrónica, como los valores de las variables del proceso que se están ejecutando en el equipo. La lógica de supervisión se ejecuta en una placa con microprocesador separado, con el objetivo de robustecer el funcionamiento del dispositivo, relajar la exigencia de procesamiento en el uC central y mantener una filosofía de operación simple. 
Innovación y Desarrollo Tecnológico y Social (2021) 3 (1): 45-88

Un circuito de watchdog (WDT), se encarga de verificar que el proceso principal del uC central, refrescando periódicamente la señal de entrada WDI (Watchdog Input). En el caso en que la señal no se encuentre presente dentro del tiempo de supervisión configurado, el WDT reseteará al uC central y ejecutará la alarma de WDT.

La UPS, que alimenta al equipo y provee de la autonomía necesaria para mantener la alimentación durante un corte de energía eléctrica, posee su propio sistema de alarma que también es monitoreado desde el circuito de supervisión.

Desde el punto de vista funcional, ver Figura 7, el supervisor se encuentra en el inicio en el estado MONITOR. En caso de recibir una interrupción, causada por el disparo de alguna de las señales de alarma ante un fallo, el sistema pasa al estado ALARM, donde permanecerá hasta que el sistema pueda ser restablecido. En el estado ALARM, se activan las señales sonoras y lumínicas para alertar al personal de salud. Se mantendrá en este estado en la medida en que ninguna de las señales haya recuperado valores nominales y normales de funcionamiento.

Si la falla puede ser resuelta, el proceso pasa al estado RESET, apagando las señales sonoras y lumínicas, para pasar al estado MONITOR nuevamente. 
Innovación y Desarrollo Tecnológico y Social (2021) 3 (1): 45-88

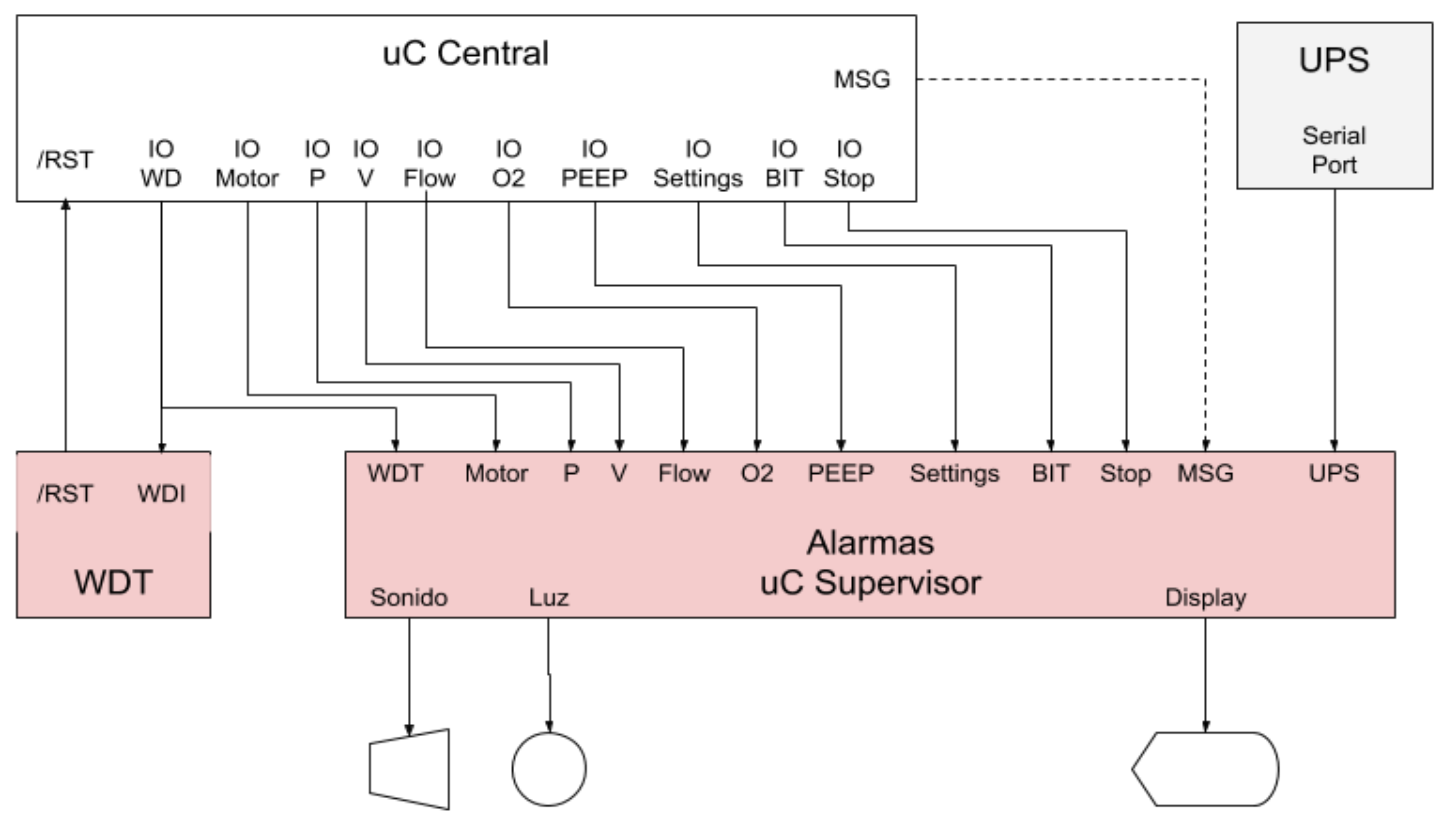

Figura 6: Señales del circuito de supervisión.

Figure 6: Supervisory circuit signals.

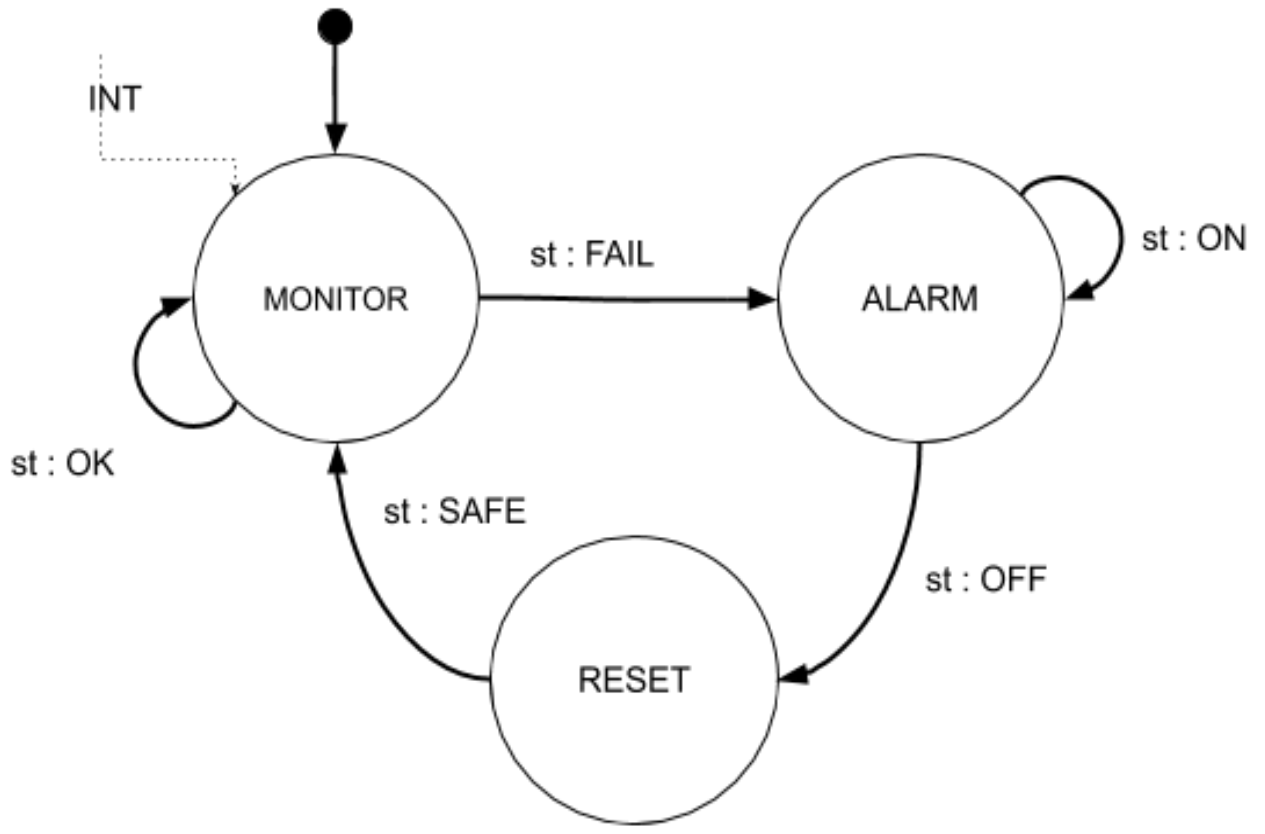

Figura 7: Máquina de estados del supervisor.

Figure 7: Supervisor state machine. 
Innovación y Desarrollo Tecnológico y Social (2021) 3 (1): 45-88

Modelo Mecánico del Sistema Respiratorio

El modelo implementado en Matlab se basa en el descripto en Levitsky (2003) y Rideout (1991). Además de las implementaciones de los modelos realizados por el Proyecto Physiome del Department of Bioengineering, University of Washington, Seattle, USA. Toma en cuenta el flujo de aire desde la boca, donde se coloca un estímulo producido por un ventilador con presión positiva. Se considera un pulso de entrada de presión ideal, con tiempo de subida nulo y se tiene en cuenta la presión PEEP. La mecánica del Sistema Respiratorio puede representarse aproximadamente por un sistema lineal invariante en el tiempo (SLIT) descripto por ecuaciones lineales a coeficientes constantes. Para una mejor comprensión puede realizarse un símil con un circuito eléctrico de parámetros concentrados (Figura 8).

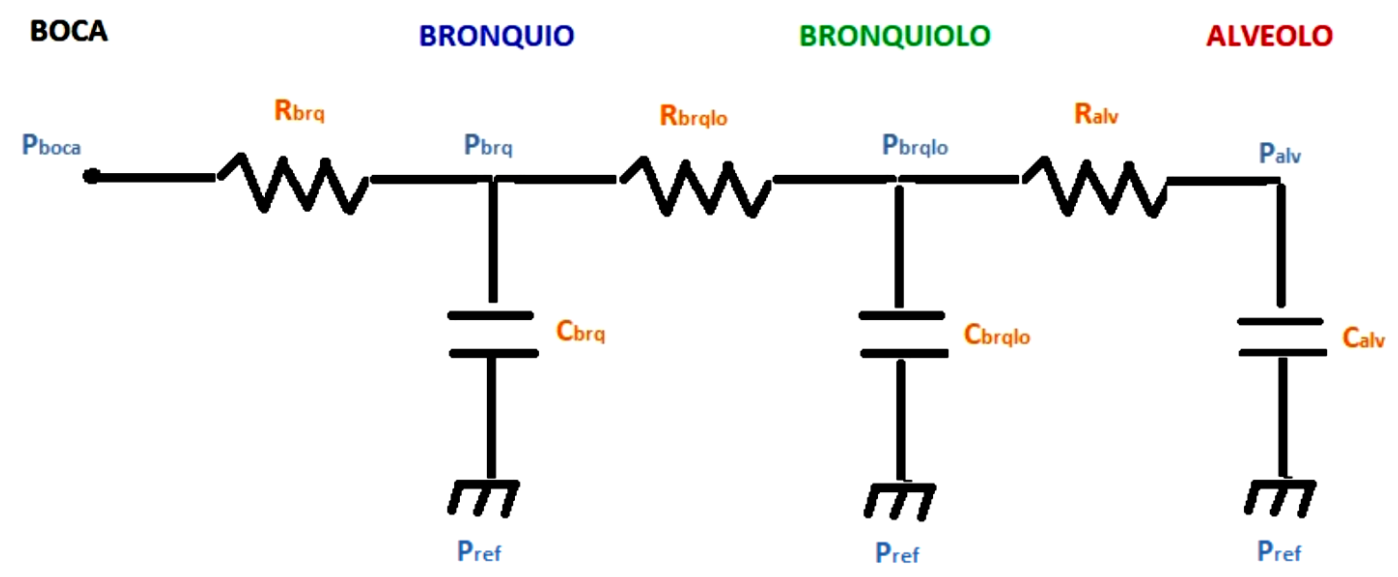

Figura 8: Equivalente eléctrico del sistema respiratorio.

Figure 8: Electrical and respiratory system equivalence. 
Innovación y Desarrollo Tecnológico y Social (2021) 3 (1): 45-88

En el modelo se representan las resistencias al pasaje de aire para los bronquios, bronquiolo y alveolo (se desprecia el efecto de la cavidad bucal, la laringe y la tráquea). La unidad para las $\mathrm{R}$ es $[\mathrm{mmHg} \mathrm{s} / \mathrm{ml}$. También se tienen en cuenta las compilancias C, que representan la capacidad de almacenar y entregar energía por elasticidad de los órganos (tejidos) que componen cada parte del sistema respiratorio. La unidad de estas C utilizada es $[\mathrm{ml} / \mathrm{mmHg}$. Luego los valores de presión en cada punto en [mmHg] son símiles de las tensiones en los circuitos eléctricos. Es evidente que las corrientes, equivalen a lo que los médicos Ilaman flujo y que para los ingenieros son los caudales [ml/s].

Se utilizaron los siguientes valores para los parámetros:

$\%$ CONSTANTES DEL SISTEMA (V. Rideout)

Rbrq $=0.002 ; \%$ Resistencia del bronquio $[\mathrm{mmHg} \mathrm{s} / \mathrm{ml}]$

Rbrqlo $=0.002 ; \%$ Resistencia del bronquiolo $[\mathrm{mmHg} \mathrm{s} / \mathrm{ml}]$

Ralv $\quad=0.01 ; \%$ Resistencia en el alveolo $[\mathrm{mmHg} \mathrm{s} / \mathrm{ml}]$

Cbrq $=1 ; \%$ Compilancia del bronquio $[\mathrm{ml} / \mathrm{mmHg}]$

C_brqlo $=3 ; \%$ Compilancia del bronquiolo $[\mathrm{ml} / \mathrm{mmHg}]$

C_alv $=50 ; \%$ Compilancia del alveolo $[\mathrm{ml} / \mathrm{mmHg}]$

Vbrq_ini $=30$; \% Volumen inicial del bronquio [ml]

Vbrqlo_ini = 120; \% Volumen inicial del bronquiolo [ml]

VFRC $=3000 ; \%$ Volumen inicial en alveolo [ml]

Pref $\quad=0 ; \%$ Presión de referencia $[\mathrm{mmHg}]$; 
Innovación y Desarrollo Tecnológico y Social (2021) 3 (1): 45-88

Para el estímulo de presión impuesto por el ventilador en boca, se utilizaron los siguientes parámetros:

di $\quad=2 ; \%$ duración de la inspiración [s]

de $\quad=2.5 ; \%$ duración de la expiración [s]

peep $=10 ; \%$ positive end expiratoty presure [\% de ampl estímulo]

Ejecutando la función considerando un estímulo perfecto del ventilador como el que muestra la Figura 9.

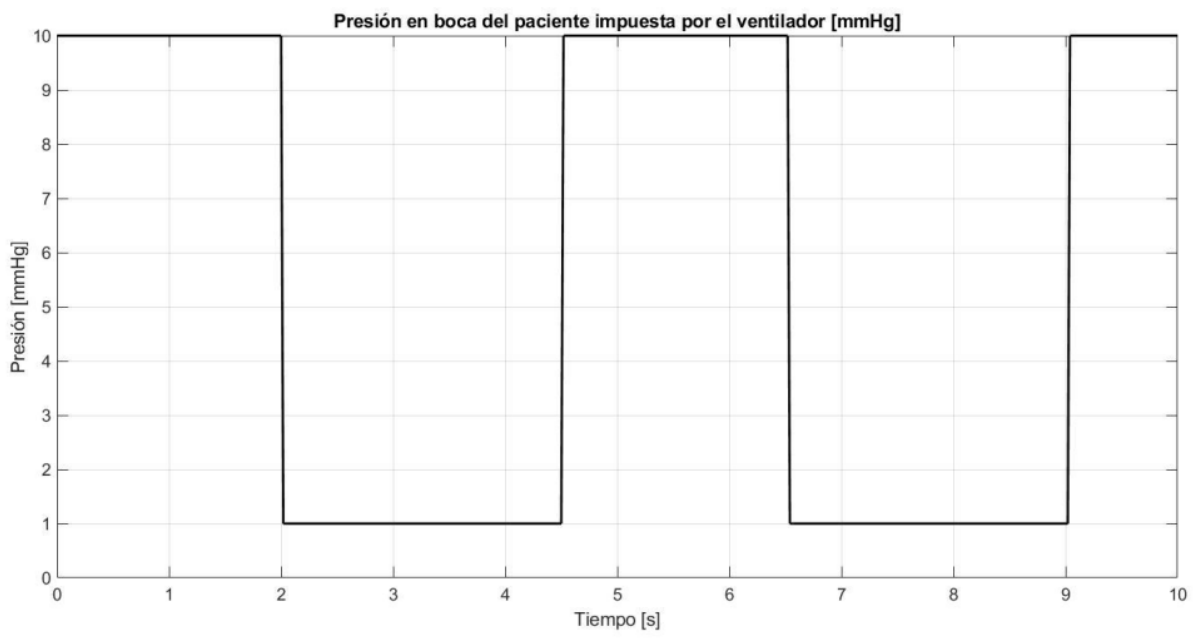

Figura 9: Presión en la boca del paciente, impuesta por el ventilador.

Figure 9: Pressure at the patient port impose by the ventilator.

Se observa el valor de la PEEP del 10\% de la amplitud máxima del estímulo, como estaba indicado en los parámetros del estímulo del ventilador. Las presiones en los distintos puntos del sistema se muestran en la Figura 10. Mientras que el volumen de aire se ve en la Figura 11 y el caudal en la Figura 12. 
Innovación y Desarrollo Tecnológico y Social (2021) 3 (1): 45-88

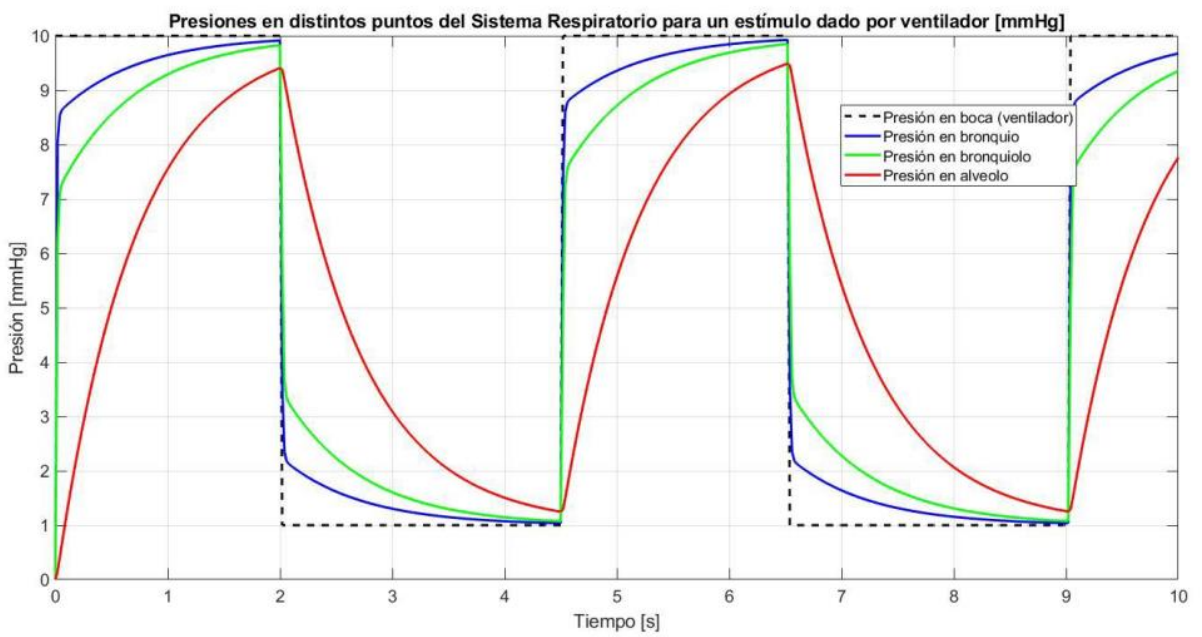

Figura 10: Presiones en distintos puntos del sistema respiratorio.

Figure 10: Pressures at different points of the respiratory system.

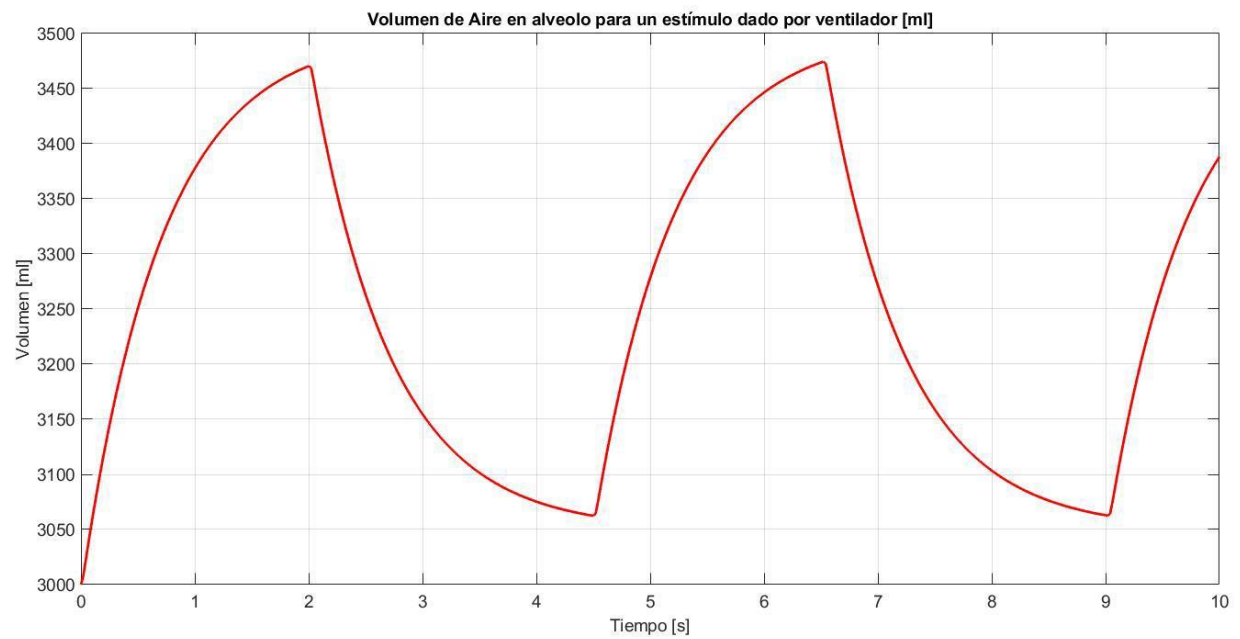

Figura 11: Volumen de aire en el alvéolo.

Figure 11: Air volume in the alveoli 


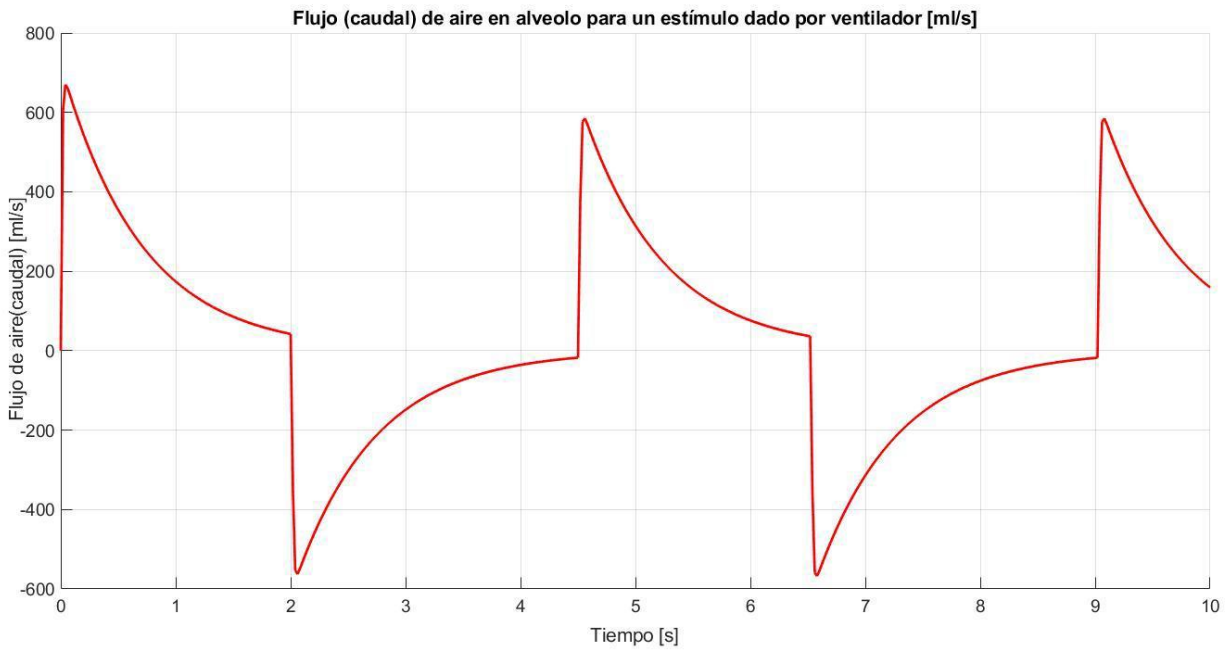

Figura 12: Flujo de aire en el alvéolo.

Figure 12: Air flow in the alveoli.

\section{Prototipo demostrador}

Teniendo en cuenta el aislamiento social obligatorio decretado por el gobierno nacional y el impacto temporal que esto implica para la adquisición de componentes, sumado al bajo presupuesto y a la escasa experiencia que el equipo de trabajo posee sobre los dispositivos de ventilación mecánica, se propuso partir de un prototipo para la verificación y validación del funcionamiento básico de los diferentes modos ventilatorios. Esto permitió volcar los requerimientos mínimos esenciales de manera temprana y validar la funcionalidad del equipo en conjunto con el propio desarrollo teórico.

Si bien responde a la funcionalidad básica, requerida para hacer ensayos funcionales, se ha construido con componentes comerciales e industriales, sin 
Innovación y Desarrollo Tecnológico y Social (2021) 3 (1): 45-88

biocompatibilidad. Por ende, no es escalable y no cumple con los requerimientos normativos impuestos por la ANMAT.

Sin embargo, esto sirvió como entrenamiento, ganando tiempo y experiencia ante la emergencia sanitaria, al tiempo que se avanzó en la búsqueda de proveedores de componentes y partes de fabricación nacional y se mitigaron los problemas de compras ocasionados por el aislamiento obligatorio. De esta manera, el diseño evolucionó hacia un segundo prototipo alfa, donde se contemplan los ensayos y las normativas impuestas para equipos médicos.

Con este prototipo se ganó experiencia en cuatros aspectos principales:

- $\quad$ El circuito mecánico y neumático.

- $\quad$ Los modos ventilatorios implementados.

- $\quad$ El diseño electrónico.

- $\quad$ La implementación del diseño de software.

En la Figura 13 se puede ver el demostrador tecnológico desarrollado en la primera etapa. En la Figura 14 se presenta el ensamble electrónico-mecánico del demostrador. 


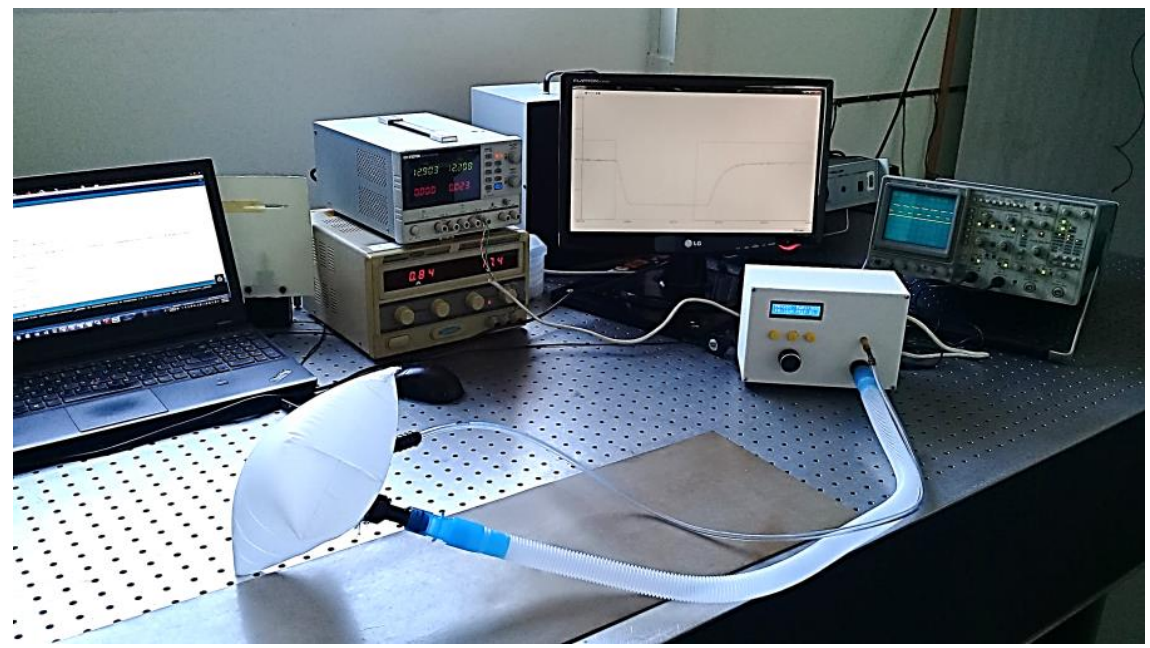

Figura 13: Modelo demostrador construido.

Figure 13: Built demonstrator model.

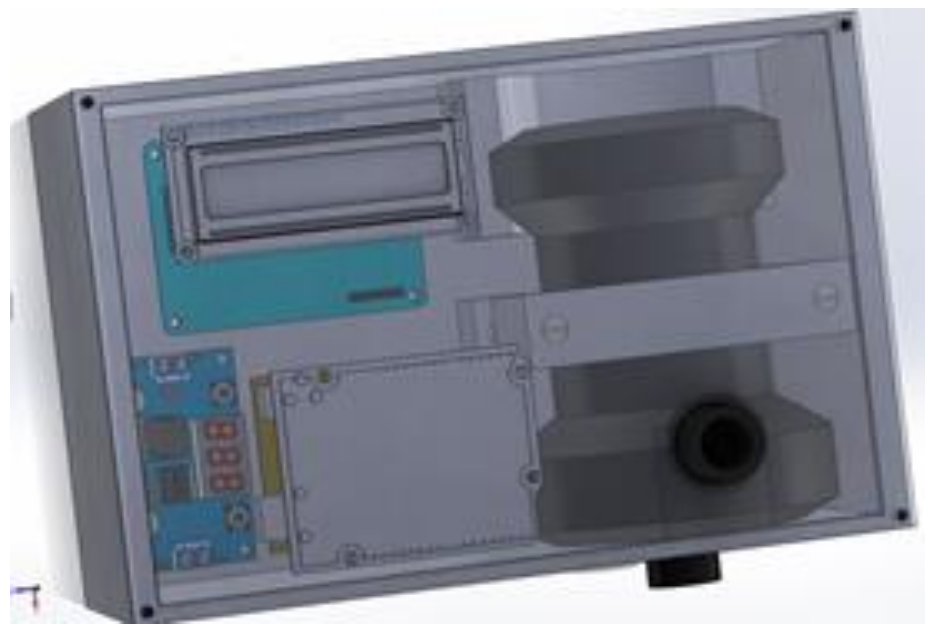

Figura 14: Ensamble electrónico-mecánico.

Figure 14: Electronic-mechanical assembly.

\section{Análisis de los ensayos y simulaciones}

Se contrastaron las señales de entrada entre el modelo demostrador y el de simulación: tensión de alimentación de la turbina y la presión de en el puerto de 
Innovación y Desarrollo Tecnológico y Social (2021) 3 (1): 45-88

salida del ventilador. Esta señal se toma como entrada del modelo lineal del sistema respiratorio más la tubuladora que conforma el circuito respiratorio. De esta manera se puede obtener la variación de presión, flujo y volumen en los alvéolos a partir de la variación de la excitación al motor de la turbina.

La Figura 15 muestra los valores para amplitud de la onda de alimentación del motor BLDC de 90\% Vcc, 75\% Vcc y 50\% Vcc (Vcc = 12v) y la respuesta de presión de salida correspondiente. Dado que para los sensores de presión no era posible medir presiones por debajo de $13 \mathrm{cmH} 2 \mathrm{O}$, se llevaron los valores de esa presión a cero. Esto último, además de modificar la señal de la respuesta (se podría interpolar o filtrar pasabajos) deja un retardo aparente de mayor duración en el contraste de los resultados.

Está a la vista que la ganancia en continua (en $\mathrm{f}=0$ ) del sistema no es constante, por lo que puede decirse que el sistema no cumple con la linealidad, aunque observamos que la respuesta al escalón de tensión es típica de un sistema de primer orden, considerando finalmente al sistema no lineal.

Se realiza la identificación como un sistema lineal, dejando como consecuencia una ganancia en continua según el par entrada-salida considerado. 
Innovación y Desarrollo Tecnológico y Social (2021) 3 (1): 45-88
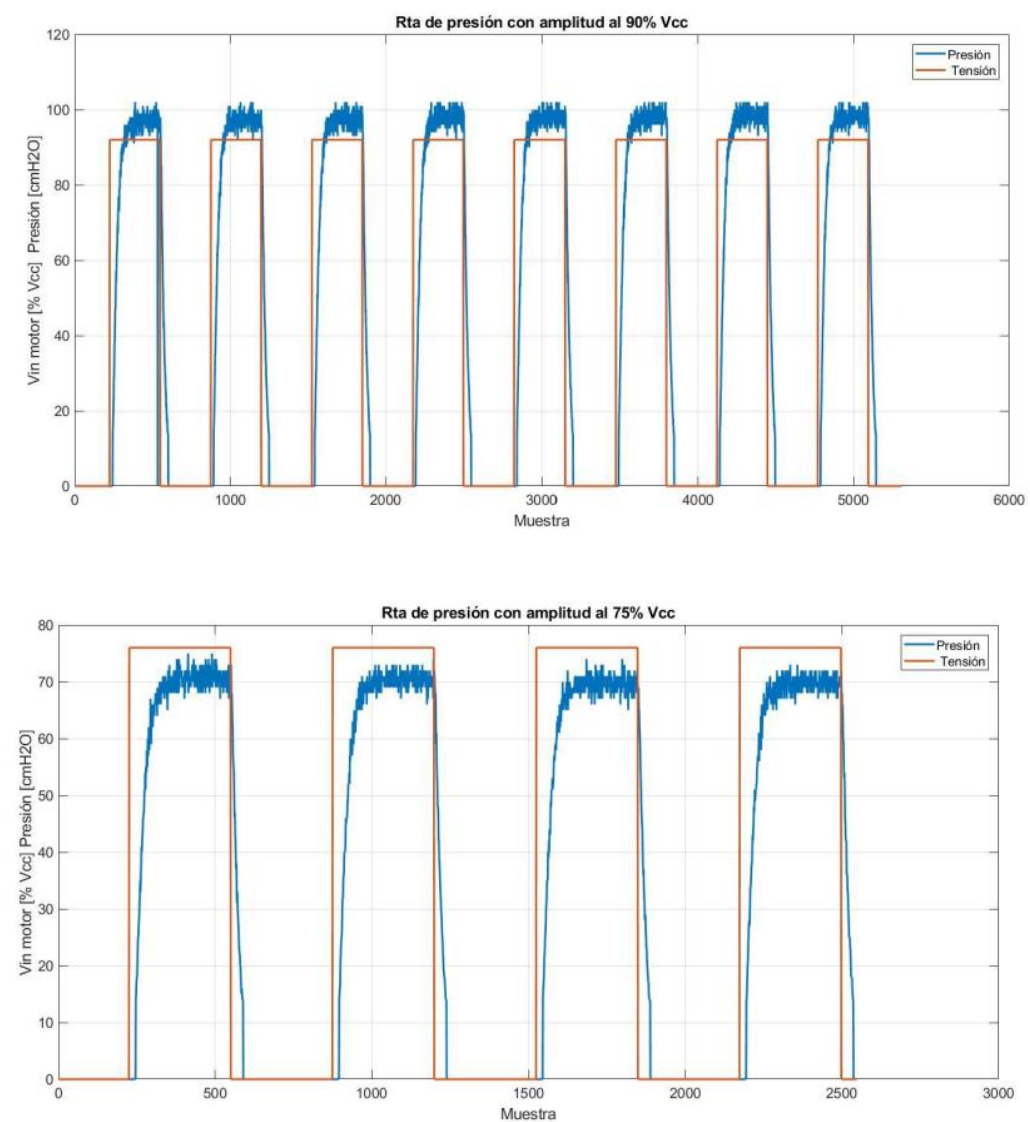

b)

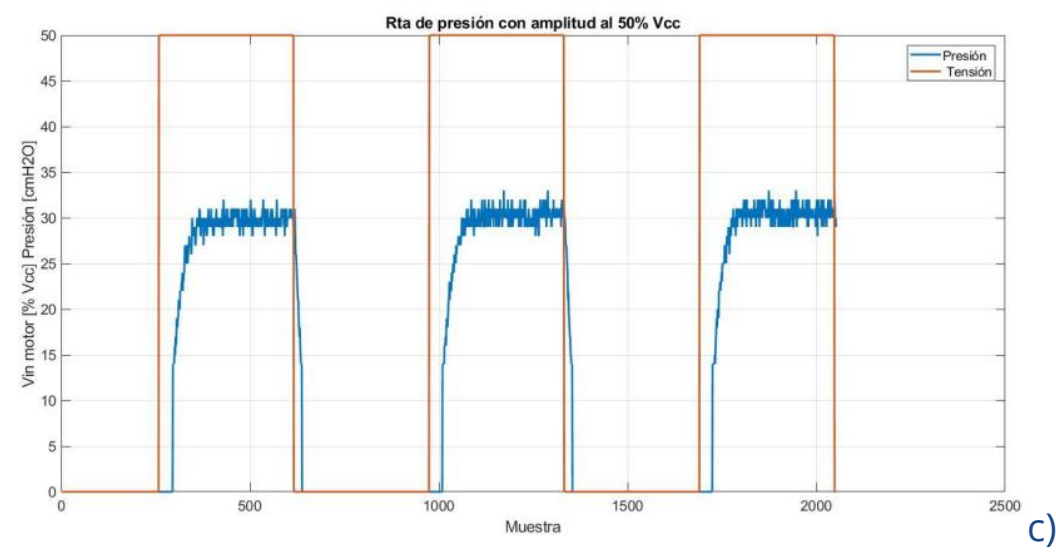

Figura 15: Presión controlada por tensión, con valores variables. a) Vcc $=90 \%$, b) Vcc $=$ $75 \%$, c) Vcc $=50 \%$.

Figure 15: Voltage controlled pressure with varying values. a) Vcc $=90 \%$, b) Vcc $=75 \%$, c) $\mathrm{Vcc}=\mathbf{5 0} \%$. 
Innovación y Desarrollo Tecnológico y Social (2021) 3 (1): 45-88

Inicialmente consideremos un SLIT de VIC con un polo único. Para el par entradasalida del 50\% de Vcc se obtiene la gráfica de la Figura 16.

Si ingresamos al sistema identificado como tf1 (por transfer function) la señal de tensión de alimentación al BLDC al 50\%, obtenemos la señal de salida que puede compararse con la original. Cabe destacar que hay diferencias más notorias, debido al cero forzado de la señal de presión de salida por debajo del límite de medición del sensor.

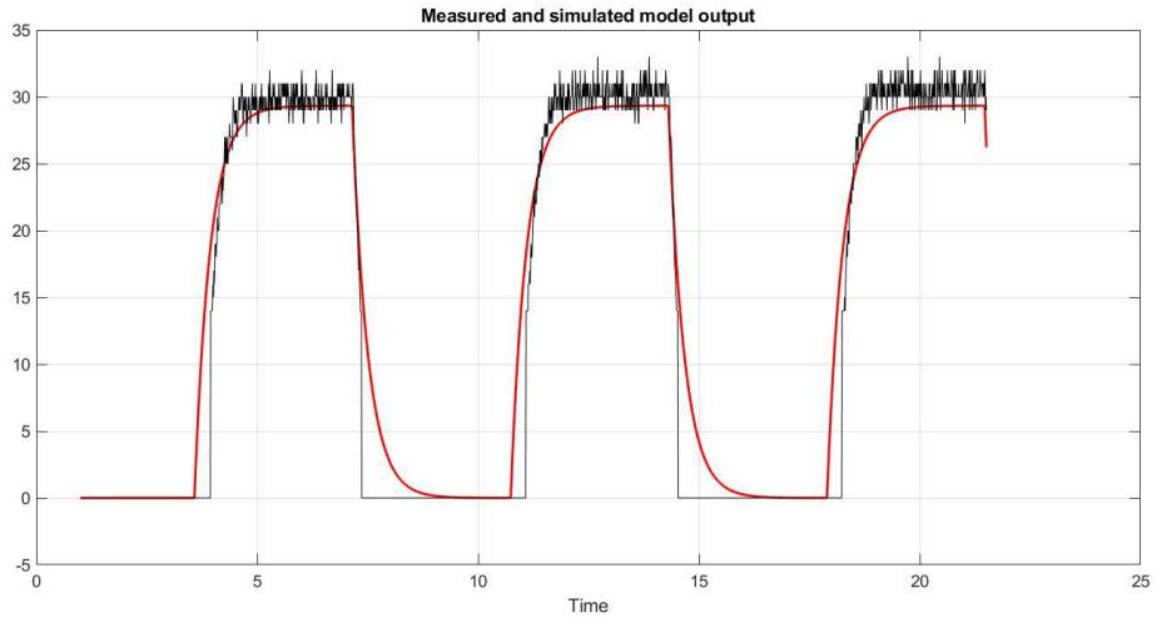

Figura 16: Salida del modelo simulada y medida con Vcc $=50 \%$.

Figure 16: Measured and simulated model output, with Vcc $=50 \%$.

Se repitió el procedimiento para la señal al $75 \%$ y $90 \%$, con la identificación del mismo modelo. La ganancia varía en continua y el polo, se ubicó en la frecuencia aproximada $\mathrm{f}=3 / 2 \mathrm{pi} \mathrm{Hz}$. Con este modelo se capturó la dinámica en alta frecuencia del sistema. 
Innovación y Desarrollo Tecnológico y Social (2021) 3 (1): 45-88

La salida de los tres modelos de sistema, a la entrada al 90\% se muestra en la Figura

17. Se aprecian diferencias entre ellos, debido al valor de ganancia en continua de cada uno.

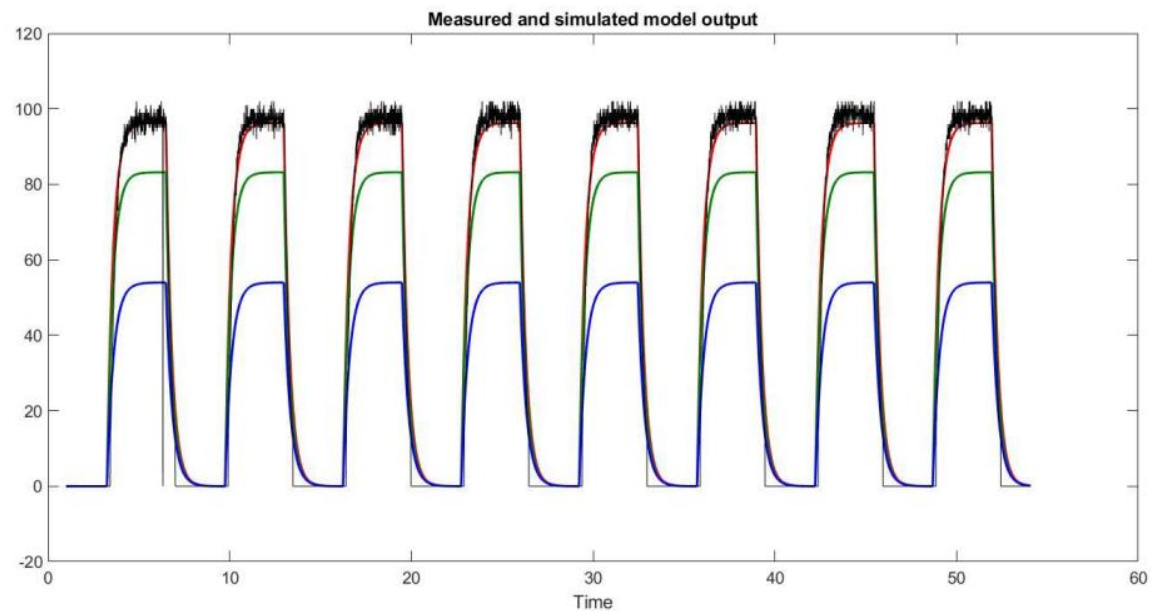

Figura 17: Salida de los 3 modelos con Vcc $=90 \%$.

Figure 17: Outputs for the 3 models, with Vcc $=90 \%$.

Posteriormente, se consideró a la planta como a un sistema lineal y concentrar las no-linealidades en dos bloques de entrada y salida. Se utilizó entonces la estimación de sistemas no lineales, con el modelo de Hammerstein-Wiener.

La salida del sistema no-lineal estimado a las tres entradas comparadas con la salida real, puede verse en la Figura 18. 
Innovación y Desarrollo Tecnológico y Social (2021) 3 (1): 45-88

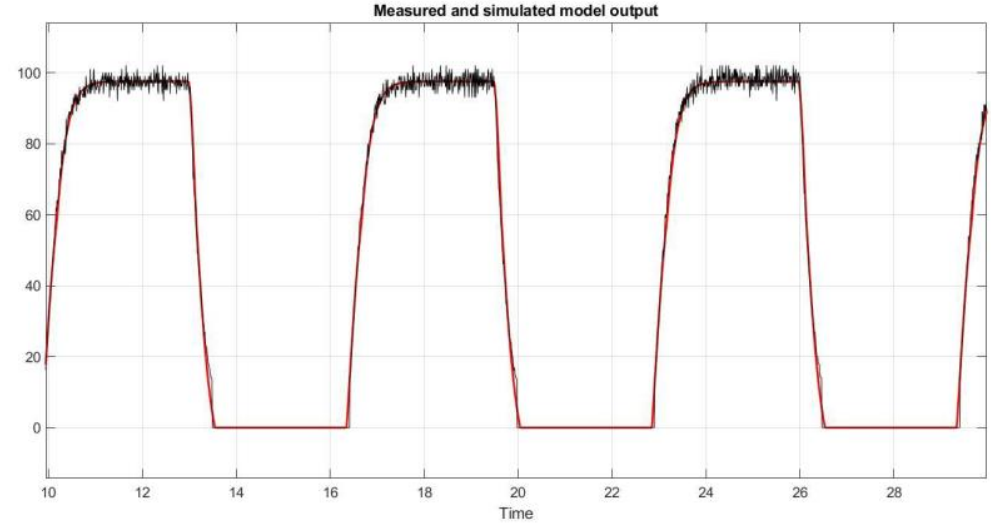

a)

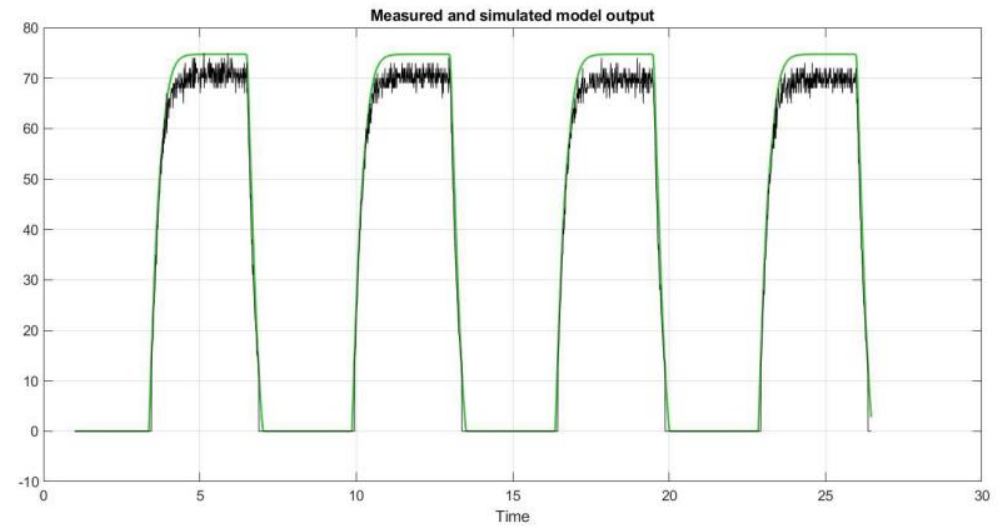

b)

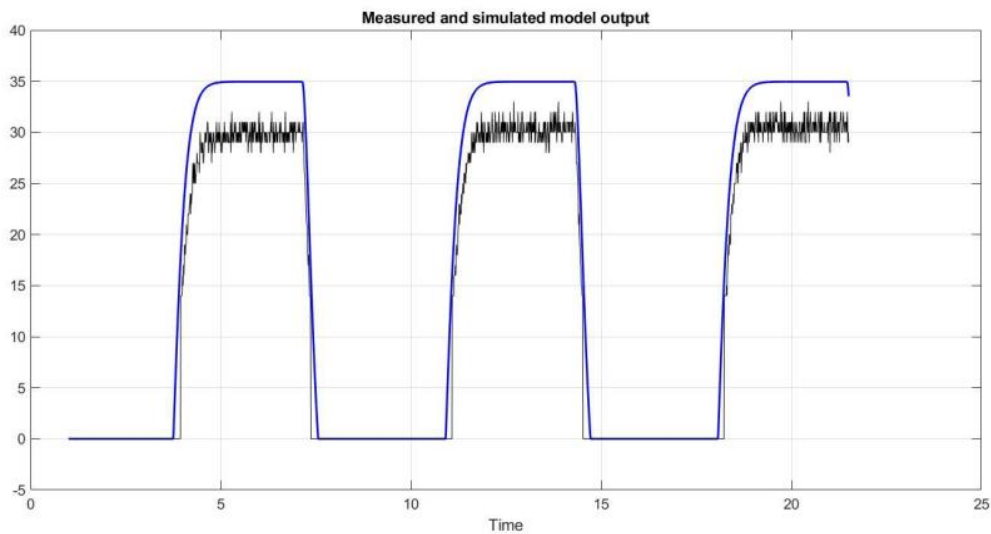

c)

Figura 18: Salidas para el modelo no lineal estimado. a) Vcc $=90 \%$, b) Vcc $=75 \%$, c) Vcc $=50 \%$.

Figure 18: Outputs for the non-linear estimated model. a) Vcc $=90 \%$, b) Vcc $=75 \%, c)$ $\mathrm{Vcc}=\mathbf{5 0} \%$. 
Innovación y Desarrollo Tecnológico y Social (2021) 3 (1): 45-88

\section{Conclusiones}

Se pudo observar que el modelo de segundo orden sobre-amortiguado (dos polos reales y un polo) representa con una precisión aceptable la dinámica del sistema tensión-presión. Aún queda pendiente la identificación final el modelo del motor BLDC del VMNI de uso médico y estimar sus parámetros para tener un buen modelo. Luego, realizar el control PI o PID basado en los modelos identificados para garantizar un correcto suministro de presión al paciente en función de las diferentes variables del sistema.

El modelo de demostración construido permitió profundizar el conocimiento de la dinámica respiratoria, en conjunto con los diferentes parámetros que conforman al VMNI y su impacto en el paciente.

La iniciativa tomada por el IAR, en colaboración con otras instituciones, empresas y particulares resultó en una experiencia única con un fuerte impacto en las actividades desarrolladas por el Instituto, con el objetivo de aportar soluciones concretas al sistema de salud nacional.

\section{Agradecimientos}

Agradecemos el apoyo del CCT La Plata, CONICET, y en especial a su Directora la Dra. Gloria Chicote, así como a los sectores de Prensa y Vinculación de esa institución. También agradecemos a Gonzalo Márquez y María Clara Lima de la UNLP por su ayuda y asesoramiento. Un agradecimiento especial para la Concejala 
Ana Herran Castagneto que impulsó la declaración del proyecto como de interés municipal.

Al desinteresado aporte del Dr. Miguel Borruel, personal del Policlínico Bancario: Dr. Miguel Ángel Chaves Zambrano, Jefe del Servicio de Terapia Intensiva, Médico de planta de la división de Terapia Intensiva Hospital de Clínicas; Lic. Lucas R. Cassani, Jefe de Kinesiologia POBA; Prof. Lic. Jonathan Fortunato, Docente Fundación H.A. Barceló; y Dr. Dante David Quiroga, Jefe de Emergencias. Dr. Miguel Fernández, intendente de Trenque Lauquen, Ing. Martin Benítez de Veng S.A., al Bioing. Carlos Fliger, Alejandro Massa Subsecretaría de CyT del Ministerio de Producción de la PBA, miembros de ADIMRA: Ing. Ignacio Venutolo, Matías Fernández.

A nuestros compañeros, quienes dedicaron su limitado tiempo para que este proyecto naciera y llegara a materializarse: Prof. Ing. Adrián Carlotto, con los modelos matemáticos, simulaciones y visión de ingeniería; futuro Ing. Gastón Valdez, con el desarrollo en tiempo record del código fuente del sistema de control; Dr. Manuel Fernandez y Téc. Eliseo Diaz, condensando la literatura para extraer los principios de funcionamiento, requerimientos y metodologías asociadas a los ventiladores; Ing. Emiliano Rasztosky y Téc. Pablo Alarcón, con el proceso de dimensionamiento, selección y compras de piezas clave en el equipo; y Ezequiel Colombo, con su experiencia en el mantenimiento y gestión de equipos médicos. También destacamos el compromiso y colaboración, aportando su tiempo y elementos para avanzar con el diseño y prototipo, a las empresas Electromedik S.A. 
por medio de Fabián Ferrario, y MBMED S.A. a través de Prof. Dr. Ing. Matías Madorno y Bioing. Paula Celoria.

\section{Referencias bibliográficas}

0221.com.ar. (1 de abril de 2020). Coronavirus: científicos platenses y de la UNLP desarrollan respiradores artificiales. 0221.com.ar. https://www.0221.com.ar/nota/2020-4-1-8-29-0-coronavirus-cientificosplatenses-y-de-la-unlp-desarrollan-respiradores-artificiales

ACUTE-19. (2020). Open Source Smart Breathing System. http://acute19.com/

Agencia Télam. (1 de abril de 2020). El Conicet desarrolla respiradores artificiales para atender casos de coronavirus. Agencia TELAM. https://www.telam.com.ar/notas/202004/446730-el-conicet-desarrollarespiradores-artificiales-para-atender-casos-de-coronavirus.html

Al Husseini, A. M., Lee, H. J., Negrete, J., Powelson, S., Servi, A. T., Slocum, A. H., y Saukkonen, J. (2010). Design and Prototyping of a Low-Cost Portable Mechanical Ventilator. Journal of medical devices, 4(2), 027514. https://doi.org/10.1115/1.3442790

América TV. (6 de abril de 2020). Así son los respiradores de bajo costo para enfrentar al coronavirus [Archivo de video]. YouTube. https://youtu.be/PhdzBfDrBX4 
Innovación y Desarrollo Tecnológico y Social (2021) 3 (1): 45-88

BA Productivo. (2020). Desarrollan en el IAR La Plata un ventilador a bajo costo para atender

el

COVID-19.

$B A$

Productivo.

https://www.baproductivo.com.ar/desarrollan-en-el-iar-la-plata-un-

ventilador-a-bajo-costo-para-atender-el-covid-19/

Diario El Día. (8 de abril de 2020). Expertos platenses desarrollan respiradores artificiales de bajo costo para darle pelea al virus. Diario El Día. https://www.eldia.com/nota/2020-4-8-2-25-20-expertos-platenses-

desarrollan-respiradores-artificiales-de-bajo-costo-para-darle-pelea-al-virus-

la-ciudad

Diario Perfil. (5 de abril de 2020). Diseñan un innovador dispositivo de asistencia respiratoria no invasivo.

Diario Perfil. https://www.pressreader.com/article/281964609831068

ECO medios | Entrevistas. (21 de abril de 2020). Gustavo Romero- Dir del Instit. Arg. de Radioastronomía @laurasverdlick 21-4-2020 [Archivo de audio]. Mixcloud. https://www.mixcloud.com/ecomedios/gustavo-romero-dir-del-instit-argde-radioastronomía-laurasverdlick-21-4-2020/

Ferrero R, G. (2008). XII Modos ventilatorios en ventilación no invasiva. Revista chilena de enfermedades respiratorias, 24(3), 240-250. https://dx.doi.org/10.4067/S0717-73482008000300012 
Innovación y Desarrollo Tecnológico y Social (2021) 3 (1): 45-88

Garmendia, O., Rodríguez-Lazaro, M. A., Otero, J., Phan, P., Stoyanova, A., Dinh-Xuan, A. T., Gozal, D., Navajas, D., Montserrat, J. M., y Farré, R. (2020). Low-cost, easyto-build noninvasive pressure support ventilator for under-resourced regions: open source hardware description, performance and feasibility testing. The European respiratory journal, 55(6), 2000846. https://doi.org/10.1183/13993003.00846-2020

IAR Extensión. (26 de abril de 2020). Ministro Roberto Salvarezza menciona al IAR en la Cámara de Diputados [Archivo de video]. YouTube. https://youtu.be/Nn $5 \mathrm{HxrGHk}$

Investiga UNLP. (1 de abril de 2020). Investigadores de la UNLP, en el desarrollo de respiradores de bajo costo. Investiga.unlp.edu.ar. https://investiga.unlp.edu.ar/cienciaenaccion/investigadores-de-la-unlp-enel-desarrollo-de-respiradores-de-bajo-costo-17691

Lee, J. (2020). Low-cost open-source ventilator device. https://github.com/jcl5m1/ventilator

Levitsky, M.G. (2003). Pulmonary Physiology. Sixth Edition, McGraw Hill.

Makers-for-life. (2020). MakAir Covid-19 Ventilator. https://github.com/makers-forlife/makair

NIOSH. (1996). NIOSH Guide to the Selection and Use of Particulate Respirators. 
Innovación y Desarrollo Tecnológico y Social (2021) 3 (1): 45-88

DHHS

$(\mathrm{NIOSH})$

Publication

Number

96-101.

https://www.cdc.gov/niosh/docs/96-101/default.html

Pennisi, D. (2020). Arduino PRO Addressing COVID-19 challenges. https://drive.google.com/file/d/1uWDG-2Wc8ZobGBOS5fWSWNvOp-

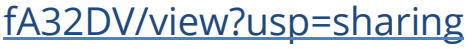

Radio Nacional. (1 de abril de 2020). COVID 19: Expertos del Conicet desarrollan respiradores artificiales. Radio Nacional. http://www.radionacional.com.ar/covid-19-expertos-del-conicetdesarrollan-respiradores-artificiales/

Rideout, V.C. (1991) Mathematical and Computer Modeling of Physiological Systems. Prentice Hall, p137-143.

SADI. (20 de marzo de 2020). Tratamiento de Sostén actualizado al 19 de marzo. Ministerio de Salud de la Nación - Sociedad Argentina de Terapia Intensiva. https://www.sadi.org.ar/rss/item/948-covid-19-tratamiento-de-sosten

SATI. (2020). Mínimos clínicamente aceptables para el desarrollo de dispositivos capaces de dar soporte ventilatorio durante la pandemia generada por COVID19. Novedades de la Sociedad Argentina de Terapia Intensiva. https://www.sati.org.ar/images/SATI_Minimos_clinicamente_aceptables_par a el desarrollo de dispositivos capaces de dar soporte ventilatorio dura 
Innovación y Desarrollo Tecnológico y Social (2021) 3 (1): 45-88

\section{nte_la_Pandemia_generada_por_COVID_19.pdf}

SEMES. (2020). Recomendación asistencia y soporte respiratorio Covid-19 (2019$\mathrm{nCoV}$ ) en transporte sanitario terrestre. Sociedad Española de Medicina de Urgencias y Emergencias. Versión 1.2-2. https://www.semes.org/wpcontent/uploads/2020/03/Recomendacio\%CC\%81n-asistencia-y-soporterespiratorio-COVID-19-transporte-terrestre-V1.2-2.pdf

Thomas, A. N., y Galvin, I. (2008). Patient safety incidents associated with equipment in critical care: a review of reports to the UK National Patient Safety Agency. Anaesthesia, 63(11), 1193-1197. https://doi.org/10.1111/j.13652044.2008.05607.x

TN noticias. (1 de abril de 2020). Coronavirus: expertos del Conicet desarrollan respiradores artificiales. $T N$ noticias. https://tn.com.ar/salud/noticias/2020/04/01/coronavirus-expertos-delconicet-desarrollan-respiradores-artificiales/

TV Universidad. (8 de junio de 2020). \#Expertos UNLP - Ingenieros del IAR crean respiradores no invasivos para combatir la pandemia [Archivo de video]. YouTube. https://youtu.be/7is0DhcYkD4 